\title{
A new topological structure for the Langevin-type ultrasonic transducer
}

\author{
Xiaolong Lu, Junhui Hu*, Hanmin Peng, Yuan Wang
}

\begin{abstract}
:
In this paper, a new topological structure for the Langevin-type ultrasonic transducer is proposed and investigated. The two cylindrical terminal blocks are conically shaped with four supporting plates each, and two cooling fins are disposed at the bottom of terminal blocks, adjacent to the piezoelectric rings. Experimental results show that it has larger vibration velocity, lower temperature rise and higher electroacoustic energy efficiency than the conventional Langevin transducer. The reasons for the phenomena can be well explained by the change of mass, heat dissipation surface and force factor of the transducer. The proposed design may effectively improve the performance of ultrasonic transducers, in terms of the working effect, energy consumption and working life.
\end{abstract}

Keywords: ultrasonic transducer; high-performance; topological structure 


\section{Introduction}

The development of various applications of power ultrasound requires ultrasonic transducers with larger maximum vibration velocity and energy-efficiency, and lower temperature rise [1-3]. Larger maximum vibration velocity leads to stronger acoustic pressure in liquid, and thus the acoustic cavitation effect will be strengthened [4-5]. High energy efficiency decreases the operation cost of sono-reactors [6]. Lower temperature rise in the transducers increases the transducer lifetime and decreases the heat transferred into the treated samples such as the biological materials [1].

Langevin transducers are often utilized to generate low-frequency and high-power ultrasound $[1,7]$. P. Langevin designed and improved the classic sandwich-type ultrasonic transducer half a century ago [8], which utilizes the terminal clamping masses to increase the maximum vibration displacement of the piezoelectric components and to increase the operation stability $[9,10]$. Since then, many designs have been concentrated on the solid horn structure (e.g. conical, exponential, stepped, et al.), which can be used to amplify the vibration displacement of Langevin transducers [11-14]. Peshkovsky et al. summarized the design principles of the ultrasonic horn based on his "five-element" theory and invented the bar-bell horn which has a large gain factor [4-6]. S. Lin analyzed the relationship between the vibration amplitude and ultrasonic transducer's geometrical dimensions and proposed the optimized methods to improve the radiation power [15-18]. Recently, J. A. Gallego-Juárez et al. proposed and investigated a radiator structure with properly designed slots for acquiring high acoustic power capacity, high efficiency and controlled radiation patterns [19-22]. Besides those, Rubio et al. applied functionally graded material technique to design piezoelectric transducers for acquiring little stress concentration and wide bandwidth [23]. Nakamura et al. designed an airborne ultrasonic transducer composed of a poly-based vibrator and high-intensity ultrasound can be generated [24].

In this work, a novel ultrasonic transducer structure, which is achieved by optimizing the mass distribution of the terminal blocks of a Langevin transducer, is reported. In the structure, the two cylindrical terminal mass blocks are conically shaped with four supporting plates each, and two cooling fins are disposed at the bottom of the terminal mass blocks, adjacent to the piezoelectric rings. A prototype is designed with the assistance of the finite element method (FEM), and 
fabricated to verify the proposed design. The results show that, at the same driving voltage and resonance, the proposed transducer has larger vibration amplitude, acoustic output power and electroacoustic energy efficiency, and lower temperature rise than the traditional Langevin transducers. Physical reasons for the transducer's improved performance are also analyzed and discussed. The transducer proposed in this work may be used to effectively improve the treatment effects, energy efficiency and lifetime of various sono-reactors.

\section{Structure design}

Fig. 1(a) shows the configuration of a traditional Langevin transducer (TLT) which consists of two terminal mass blocks (the front mass block and back mass block), one bolt and two piezoelectric rings (outer diameter $40 \mathrm{~mm} \times$ inner diameter $16 \mathrm{~mm} \times$ thickness $6 \mathrm{~mm}$ ). The components are disposed as shown in Fig. 1(a), and clamped together by the bolt. Two piezoelectric rings are stacked with opposite polarization directions in order to excite the longitude vibration mode in the axial direction. When the TLT operates at resonance frequency of the 1st order longitudinal mode, ultrasound energy is radiated from the end surface of the terminal mass block. The size of the TLT is $100 \mathrm{~mm}$ (length) $\times 40 \mathrm{~mm}$ (diameter), and the length of the terminal mass block is $44 \mathrm{~mm}$.

Lots of works in ultrasonic transducers use the tapered front mass to enhance the vibrating performance at the expanse of reducing the size of the radiation face [11-14]. Being different from those works, we propose the mass optimized transducer (MOLT) in this work, which uses the same piezoelectric rings and possesses the same radiation face size with the TLT shown in Fig. 1(a). Fig. 1(b) shows the structure of the proposed transducer. The structure difference between the TLT and MOLT is in the terminal mass blocks. As shown in Fig. 1(b), the MOLT's terminal mass blocks can be divided into three parts: part I is a circular mass plate with a radiation face which has the same area as that of the TLT; part II is a cone-shaped mass block with four supporting plates uniformly disposed on the cone surface between parts I and III; part III is a cylinder with a circular ring as heat sink.

PZT-5H, which can be used as the hard piezoelectric material for transmitting transducers [25, 26], is used in the transducer. Bronze is chosen as the electrodes, and steel for the transducer's 
other metal parts. Detailed material property constants used in the computation are listed in Table $1[27,28]$.

\section{Finite element analyses}

Vibration and thermal characteristics of the transducers have been analyzed by COMSOL MULTIPHYSICS FEM software. In the first step, the 3-dimensional transducer model was built by Pro Engineering software and then transferred into COMSOL. In the second step, material of each part of the transducers was defined. In the third step, the models were meshed into elements, and boundary conditions were applied. In the fourth step, vibration characteristics were computed by using the eigen frequency module and frequency domain module. In the last step, thermal characteristics were computed by the calculation module Heat Transfer in Solids. By the above calculating process, both the TLT and MOLT were modeled and analyzed for a comparison. In the calculation, unless otherwise specified, driving voltage applied on the Langevin transducers was $40 \mathrm{~V}_{\mathrm{p}-\mathrm{p}}$ and the initial temperature was $25^{\circ} \mathrm{C}(298 \mathrm{~K})$.

In the vibration analyses, the $1^{\text {st }}$ longitudinal vibration modes of both transducers were analyzed, and the calculated vibration modes are shown in Fig. 2, respectively. Calculated resonance frequency for the TLT is $21.3 \mathrm{kHz}$ and that for the MOLT is $22.2 \mathrm{kHz}$, which means that the new structure of the MOLT shifts the resonance frequency by $4.2 \%$. According to the calculation, it is observed that vibration amplitude of the MOLT $\left(2.5 \times 10^{-6} \mathrm{~m}\right)$ at resonance is two times higher than that of the TLT $\left(0.8 \times 10^{-6} \mathrm{~m}\right)$ also at resonance for the same voltage, which indicates that vibration magnitude is enhanced for the MOLT's structure by removing some unnecessary mass in the terminal mass blocks.

In the thermal analyses, power loss density obtained from the vibration calculation was used as the heat source for computing the temperature field distribution. Fig. 3 shows the steady state temperature field of the TLT and MOLT, respectively. The colored iso-surfaces denote the calculated temperature value, and the arrows denote the direction of heat flux. Generally speaking, piezoelectric rings are the hottest structures in both TLT and MOLT. From the TLT's result, it can also be seen that temperature difference between the maximum (307 K) and minimum values (300 $\mathrm{K}$ ) is $7 \mathrm{~K}$, and all heat in the transducer transfers to the radiation faces. Comparatively, the MOLT's temperature difference is $4 \mathrm{~K}$ and heat dissipates mainly through the cooling fins, which 
indicates that thermal characteristic is improved for the MOLT by optimizing the mass distribution.

\section{Results and discussion}

According to the above design and analyses, prototypes of the TLT and MOLT were fabricated and assembled, respectively (see Fig. 4). In the following experiments, unless otherwise specified, the driving voltage of the transducers was identical $\left(=40 \mathrm{~V}_{\mathrm{p}-\mathrm{p}}\right)$, and both transducers operate at resonance. Impedance characteristics of the transducers were measured by an impedance analyzer (Agilent 4294A), and measured results are shown in Fig. 5. Detailed electrical parameters measured by the impedance analyzer PV80A [29] are listed in Table 2.

A laser Doppler vibrometer system (PSV-300F-B) was used in the measurement of vibration characteristics of the transducers. Fig. 6(a) shows the measured out-of-plane vibration distribution of the two transducers' radiation faces. Measured resonance frequency of the TLT is $20.6 \mathrm{kHz}$ and

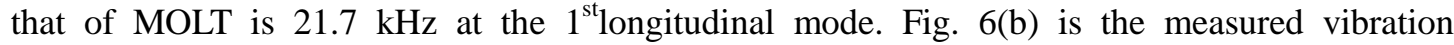
amplitude at the radiation surface versus the radial location $R$. It is seen that the vibration amplitude of the TLT changes little for $0 \mathrm{~mm} \leqslant \mathrm{R} \leqslant 20 \mathrm{~mm}$, and the vibration amplitude of the MOLT increases as $R$ increases for $R<8 \mathrm{~mm}$ and increases little for $8 \mathrm{~mm} \leqslant R \leqslant 20 \mathrm{~mm}$. It is also seen that the most part of the MOLT's radiation face has higher vibration magnitude than the TLT's radiation face, which indicates that the MOLT can radiate more acoustic energy than the TLT at the radiation face while driven by the same voltage at resonance. This phenomenon is caused by the smaller mass of the MOLT. This experiment also confirms the calculated results shown in Fig. 2. The differences between the theoretical and experimental vibration amplitudes could be caused by the clamping force and the bolt, which were simplified in the calculation model.

An infrared thermal camera (FLIR I7) was used to measure thermal characteristics of the prototypes at resonance (ambient temperature: $26^{\circ} \mathrm{C}$ ). It was found that the point with the maximum surface temperature was located at the piezoelectric rings, which agreed well with the calculated ones. Thus the point located at the middle of prototype was chosen as the temperature measurement point. Measured temperature of this point for different driving voltages and operating duration of 27 minutes are shown on the top left of each inset in Fig. 7. It is seen that the 
maximum surface temperature increases as the driving voltage increases (from $20 \mathrm{~V}_{\mathrm{p}-\mathrm{p}}$ to $60 \mathrm{~V}_{\mathrm{p}-\mathrm{p}}$ ) for both prototypes at resonance, and more importantly, the MOLT's maximum surface temperature is always a bit lower than that of the TLT under the same driven conditions and at resonance. This is because the MOLT has a larger surface area for the heat dissipation.

Measured transient-state of the maximum surface temperature of the TLT and MOLT are shown in Fig. 8. It shows that the MOLT has a lower temperature than the TLT, and reaches the stable state more quickly as the driving voltage increases. This is also because the MOLT has a larger surface area for the heat dissipation.

In order to measure the electroacoustic efficiency of the proposed prototype transducers, an experimental setup was built, as shown in Fig. 9. A hollow PVC pipe with the dimensions of 220 $\mathrm{mm}$ (length) $\times 40 \mathrm{~mm}$ (outer diameter) $\times 2 \mathrm{~mm}$ (thickness) was bonded onto the radiation face of the transducer for the measurement. $200 \mathrm{~mL}$ of water was filled into the pipe, and acted as the liquid media for receiving the radiated ultrasound power. An ultrasound power density analyzer YP0511A (Hangzhou Chenggong Ultrasonic Instruments Co. Ltd. [30]), equipped with one rod-type measurement probe, was used to measure the ultrasound power intensity in the water. The measurement probe with the terminal diameter of $8 \mathrm{~mm}$ utilizes the piezoelectric effect to measure the acoustic pressure in water, which is transferred into the electric signal and then converted to the power intensity by the power analyzer. The distance between the probe end and radiation face was $2 \mathrm{~mm}$. During the experiments, different measurement locations $L_{1}$ and $L_{2}$ were used for the measurement. Based on our measurement, the measured results were almost the same for $L_{1}$ and $L_{2}$, especially when transducers operate at high driving voltages $\left(>80 \mathrm{~V}_{\mathrm{p}-\mathrm{p}}\right)$. Thus, the position of the probe was fixed at location $L_{1}$. The acoustic output power $P_{\text {out }}$ can be calculated by the following equation:

$$
P_{\text {out }}=I_{\mathrm{p}} \cdot \pi \cdot R_{0}^{2}
$$

where $I_{\mathrm{p}}$ is the measured power intensity, and $R_{0}$ is the inner radius of the pipe. The Calorimetry method was not used in the measurement because the heat transferred from the working transducers to the water load was quite large, which caused a large measurement error.

Measured ultrasonic output power versus driving voltage for the two transducers at resonance is shown in Fig. 10(a). It can be seen that as the driving voltage increases, the ultrasonic output 
power of both transducers increases first and then decreases, and more importantly, the MOLT has a larger ultrasonic output power than the TLT, which is due to a larger vibration velocity at the radiation face of the MOLT. The TLT has a peak output power of $3 \mathrm{~W}$ at $220 \mathrm{~V}_{\mathrm{p}-\mathrm{p}}$, and the MOLT has a peak output power of $4.4 \mathrm{Watt}$ at $240 \mathrm{~V}_{\mathrm{p}-\mathrm{p}}$. When the output power of the two transducers reaches the peak values, the steady temperature rise is $10^{\circ} \mathrm{C}$ in TLT, and it is $6^{\circ} \mathrm{C}$ in MOLT.

The power density $\rho_{\mathrm{p}}$ is defined as the output power per unit mass of a transducer:

$$
\rho_{\mathrm{p}}=\frac{P_{\mathrm{out}}}{M}
$$

where $M$ is the mass of the transducer. Based on Fig. 10(a) and the mass of the TLT and MOLT ( 947 gram and 874 gram, respectively), the power density for the TLT and MOLT at resonance is $3.16 \times 10^{-3} \mathrm{~W} / \mathrm{g}$ (the steady temperature rise $=10^{\circ} \mathrm{C}$ ) and $4.12 \times 10^{-3} \mathrm{~W} / \mathrm{g}$ (the steady temperature rise $=5^{\circ} \mathrm{C}$ ), respectively, when both transducers are driven by $220 \mathrm{~V}_{\mathrm{p}-\mathrm{p}}$. This indicates that the MOLT has a higher power density under the same driving voltage and a lower temperature rise.

The electroacoustic energy efficiency $\eta$ is defined by the following equation:

$$
\eta=\frac{P_{\text {out }}}{P_{\text {in }}}=\frac{P_{\text {out }}}{\left(U_{0} \cdot I_{0} \cdot \cos \varphi\right) / 2}
$$

where $P_{\text {in }}$ is the electric input power, $U_{0}$ is the zero-to-peak value of input driving voltage, $I_{0}$ is the zero-to-peak value of input driving current, and $\varphi$ is the phase angle between the input voltage and current wave. Measured electroacoustic energy efficiency versus driving voltage of the two transducers is shown in Fig. 10(b). It is seen that the MOLT has much higher electroacoustic energy efficiency than the TLT. The force factor, which is often used in the design and analyses of ultrasonic transducers, is employed here to explain the above measured result. It is the motional current per vibration velocity.

$$
A=\frac{I_{\mathrm{m}}}{v_{0}}=\frac{U_{0} /\left|Z_{\mathrm{m}}\right|}{2 \pi f_{0} \cdot D_{0}}
$$

where $v_{0}$ is the vibration velocity, $I_{\mathrm{m}}$ is the motional current (the current in the mechanical branch of the equivalent circuit), $U_{0}$ is the driving voltage, $f_{0}$ is the resonant frequency, $D_{0}$ is the vibration amplitude at the driving voltage $U_{0}$, and $\left|Z_{\mathrm{m}}\right|$ is the impedance of motional branch of the equivalent circuit of the transducer. Using the parameters listed in Table 3, the force factor $A$ of the MOLT and TLT at $40 \mathrm{~V}_{\mathrm{p}-\mathrm{p}}$ and resonance were calculated to be $0.97 \mathrm{~A} \cdot \mathrm{s} \cdot \mathrm{m}^{-1}$ and $2.63 \mathrm{~A} \cdot \mathrm{s} \cdot \mathrm{m}^{-1}$, 
respectively. Thus the MOLT needs less motional current for obtaining the same vibration velocity. A small force of MOLT also means that its mechanical power loss is lower.

For both transducers, the electroacoustic energy efficiency increases first and then decreases as the driving voltage increases. One of reasons for this phenomenon is that as the driving voltage increases, the acoustic bubbles on the radiation face hinder the radiation of ultrasonic power [1]. The change of material properties of the transducers may also cause the phenomenon [31].

\section{Conclusions}

A new topological structure for the Langevin-type transducer has been proposed and investigated. The two cylindrical terminal blocks are conically shaped with four support plates each, and two cooling fins are disposed at the bottom of the terminal blocks, adjacent to the piezoelectric rings. The characteristics of the new transducer have been investigated by experimental measurement and FEM calculation, and compared the traditional Langevin transducer. Under the resonance condition and with the same driving voltage, the new transducer has larger vibration velocity, lower temperature rise and higher electroacoustic energy efficiency than the conventional Langevin transducer. The better performance attributes to the decrease of mass and force factor, and the increase of the heat dissipation surface. In the new transducer, the vibration velocity decreases by $50 \%$ from the outer edge of the radiation face to the center. The transducer may be further optimized, based on the requirements of detailed applications.

\section{Acknowledgements}

This work is supported by the following funding organizations in China: Fundamental Research Funds for the Central Universities (No. NJ20150002), the National Basic Research Program of China (973 Program, Grant No. 2015CB057501), National Science Foundation of China (No. 51505222), and the "111" project.

\section{REFERENCES}


[1] T.J. Mason, J.P. Lorimer, Applied sonochemistry: Uses of Power Ultrasound in Chemistry and Processing, Wiley-VCH Verlag GmbH \& Co. KGaA, 2002.

[2] T.J. Mason, Sonochemistry and sonoprocessing: the link, the trends and (probably) the future, Ultrason. Sonochem. 10 (2003) 175-179.

[3] A.S. Peshkovsky, S.L. Peshkovsky, Industrial-scale processing of liquids by high-intensity acoustic cavitation-the underlying theory and ultrasonicequipment design principles, in: F.M. Nowak (Ed.), Sonochemistry: Theory Reactions and Syntheses, and Applications, Nova Science publishers, Hauppauge, NY, 2010.

[4] S.L. Peshkovsky, A.S. Peshkovsky, Matching a transducer to water at cavitation: acoustic horn design principles, Ultrason. Sonochem. 14 (2007) 314-322.

[5] S.L. Peshkovsky, A.S. Peshkovsky, Shock-wave model of acoustic cavitation, Ultrason. Sonochem. 15 (2008) 618-628.

[6] A.S. Peshkovsky, S.L. Peshkovsky, Acoustic cavitation theory and equipment design principles for industrial applications of high-intensity ultrasound, in: Book Series: Physics Research and Technology, Nova Science Publishers, Hauppauge, NY, 2010.

[7] H. Allik, K.M. Webman, J.T. Hunt, Vibrational response of sonar transducers using piezoelectric finite elements, J. Acoust. Soc. Am. 56 (1974) 1782-1791.

[8] P. Langevin, French Patents Nr. 502913 (1920), Nr. 505703 (1920), Nr. 575435(1924).

[9] E.A. Neppiras, The prestressed piezoelectric sandwich transducer, Ultrasonics International Conf. Proc. (1973) 295-302.

[10] E. Heikkola, K. Miettinen, P. Nieminen, Multiobjective optimization of an ultrasonic transducer using NIMBUS, Ultrasonics. 44(2006) 368-380.

[11] E. Eisner, Design of sonic amplitude transformers for high magnification, J. Acoust. Soc. Am. 35 (1963) 1367-1377.

[12] E. Eisner, in: W.P. Mason (Ed.), Methods and Devices, Part B, vol. 1, Academic Press, New York, 1964.

[13] A. Bangviwat, H.K. Ponnekanti, R.D. Finch, Optimizing the performance of piezoelectric drivers that use stepped horns, J. Acoust. Soc. Am. 90 (1991)1223-1229.

[14] K. Adachi, S. Ueha, Modal vibration control of large ultrasonic tools with the use of wave-trapped horns, J. Acoust. Soc. Am. 87(1990) 208-214. 
[15] Z. Fu, X. Xian, S. Lin, C. Wang, W. Hua, G. Li, Investigations of the barbell ultrasonic transducer operated in the full-wave vibrational mode, Ultrasonics. 52 (2012) 578-586.

[16] S. Lin, Optimization of the performance of the sandwich piezoelectric ultrasonic transducer, J. Acoust. Soc. Am. 115 (2004) 182-186.

[17] S. Lin, C. Xu, Analysis of the sandwich ultrasonic transducer with two sets of piezoelectric elements, Smart Mater. Struct. 17 (2008) 065008.

[18] S. Lin, Study on the multifrequency Langevin ultrasonic transducer, Ultrasonics. 33 (1995) $445-448$.

[19] J.A. Gallego-Juárez, High power ultrasonic transducers in Sonochemistry and Sonoluminescence, in: L.A. Crum, T.J. Mason, J.L. Reisse, K.S. Suslick (Eds.),Kluwer Academic Publishers., Dordrecht, Holland, NATO ASI Series, 1999.

[20] S.A. Nayfeh, A.H. Nayfeh, Energy transfer from high to low frequency modes in a flexible structure via modulation, J. Vib. Acoust. Trans. ASME 116(1994) 203-207.

[21] A. Cardoni, M. Lucas, Enhanced vibration performance of ultrasonic block horns, Ultrasonics. 40 (2002) 365-369.

[22] J.A. Gallego-Juárez, G. Rodriguez, V. Acosta, E. Riera, Power ultrasonic transducers with extensive radiators for industrial processing, Ultrason. Sonochem. 17 (2010) 953-964.

[23] W. M. Rubio, F.Buiochi, J. C.Adamowski, E. C. N. Silva, Modeling of functionally graded piezoelectric ultrasonic transducers, Ultrasonics. 49 (2009) 484-494.

[24] J. Wu, Y. Mizuno, M.Tabaru, K. Nakamura, Airborne ultrasonic transducer using polymer-based elastomer with high output-to-weight ratio, Jpn. J. Appl. Phys. 54(2015) 087201.

[25] D. Wang, W. Chuang, K. Hsu and H. Pham, Design of a Bézier-profile horn for high displacement amplification, Ultrasonics. 51(2011) 148-156.

[26] H. Nguyen, H. Nguyen, J. Uan and D. Wang, A nonrational B-spline profiled horn with high displacement amplification for ultrasonic welding, Ultrasonics. 54(2014) 2063-2071.

[27] H. A. Kunkel, S. Locke, B. Pikeroen, Finite-element analysis of vibrational modes in piezoelectric ceramic disks, IEEE Trans. Ultrason. Ferroelect. Freq. Control 37 (1990) 316-328.

[28] E. Ando and Y. Kagawa, Finite-element simulation of transient heat response in ultrasonic transducers, IEEE Trans. Ultrason. Ferroelectr. Freq. Control 39 (1992) 432-440.

[29] http://www.bandera.com.cn/docc.htm 
[30] http://www.fycg.com/

[31] S. Li, L. Zheng, W. Cao, Determination of full set material constants of [011] poled $0.72 \mathrm{~Pb}\left(\mathrm{Mg}_{1 / 3} \mathrm{Nb}_{2 / 3}\right) \mathrm{O}_{3}-0.28 \mathrm{PbTiO}_{3}$ single crystal from one sample, Appl. Phys. Lett. 105(2014) 012901. 
Table 1 Material property constants for the ultrasonic transducers

\begin{tabular}{llll}
\hline & & PZT-5H & Steel \\
\hline Density & $\left(\mathrm{kg} / \mathrm{m}^{3}\right)$ & 7600 & 7840 \\
Young's modulus & $\left(\mathrm{N} / \mathrm{m}^{2}\right)$ & & $19.86 \times 10^{10}$ \\
Poission's ratio & & 0.31 & 0.29 \\
Damping coefficient & & $5.5 \times 10^{-4}$ & $1.09 \times 10^{-4}$ \\
Specific heat & $(\mathrm{J} / \mathrm{kg} \cdot \mathrm{K})$ & 491 & 640 \\
Thermal conductivity & $(\mathrm{W} / \mathrm{m} \cdot \mathrm{K})$ & 1.5 & 83.5 \\
Dielectric loss factor & & $0.3 \%$ & \\
Elastic stiffness constant $c_{11}$ & $\left(\mathrm{~N} / \mathrm{m}^{2}\right)$ & $12.7 \times 10^{10}$ & \\
Piezoelectric constant $d_{33}$ & $(\mathrm{pC} / \mathrm{N})$ & 593 & \\
\hline
\end{tabular}


Table 2 Electrical parameters of the equivalent circuit of the ultrasonic transducers .

\begin{tabular}{ccccc}
\hline Transducer & $C_{0}$ & $C_{1}$ & $L_{1}$ & $R_{1}$ \\
& $(\mathrm{nF})$ & $(\mathrm{nF})$ & $(\mathrm{mH})$ & $(\Omega)$ \\
\hline TLT & 2.48 & 0.45 & 136.50 & 31.60 \\
MOLT & 2.39 & 0.32 & 174.84 & 53.42 \\
\hline
\end{tabular}

$C_{0}$ is the clamping capacitance, $C_{1}$ is the motional capacitance, $L_{1}$ is the motional inductance and $R_{1}$ is the motional resistance. 
Table 3 Working Parameters of the ultrasonic transducers at $40 \mathrm{~V}_{\mathrm{p}-\mathrm{p}}$.

\begin{tabular}{ccccc}
\hline Transducer & $f_{0}$ & $\begin{array}{c}D_{0} \\
\left(\times 10^{-6} \mathrm{~m}\right)\end{array}$ & $\begin{array}{c}U_{0} \\
(\mathrm{~V})\end{array}$ & $\begin{array}{c}|Z| \\
(\Omega)\end{array}$ \\
\hline TLT & 20.6 & 0.9 & 20 & 65.9 \\
MOLT & 21.7 & 1.2 & 20 & 125.6 \\
\hline
\end{tabular}




\section{FIGURE CAPTIONS}

Fig. 1 Configuration of (a) traditional Langevin transducer (TLT), and (b) mass optimized Langevin transducer (MOLT).

Fig. 2 Calculated vibration distribution for the transducers.

Fig. 3 Calculated temperature distribution for the transducers.

Fig. 4 Prototype transducers.

Fig. 5 Measured impedance characteristics of the transducers. (a) TLT and (b) MOLT.

Fig. 6 Measured out-of-plane vibration modes of radiation surfaces of the transducers. (a) Vibration distribution. (b) Vibration amplitude versus radial location $R$.

Fig. 7 Measured temperature fields of two prototype transducers at different driving voltages. (a) TLT and (b) MOLT.

Fig. 8 Temperature rise versus operating time at different driving voltages. (a) $20 \mathrm{~V}_{\mathrm{p}-\mathrm{p}}$, (b) $40 \mathrm{~V}_{\mathrm{p}-\mathrm{p}}$ and (c) $60 \mathrm{~V}_{\mathrm{p}-\mathrm{p}}$.

Fig. 9 Experimental setup to measure the electroacoustic efficiency versus driving voltage.

Fig. 10 Measured electroacoustic efficiency versus driving voltage. (a) Acoustic output power versus driving voltage, (b) Electroacoustic efficiency versus driving voltage. 


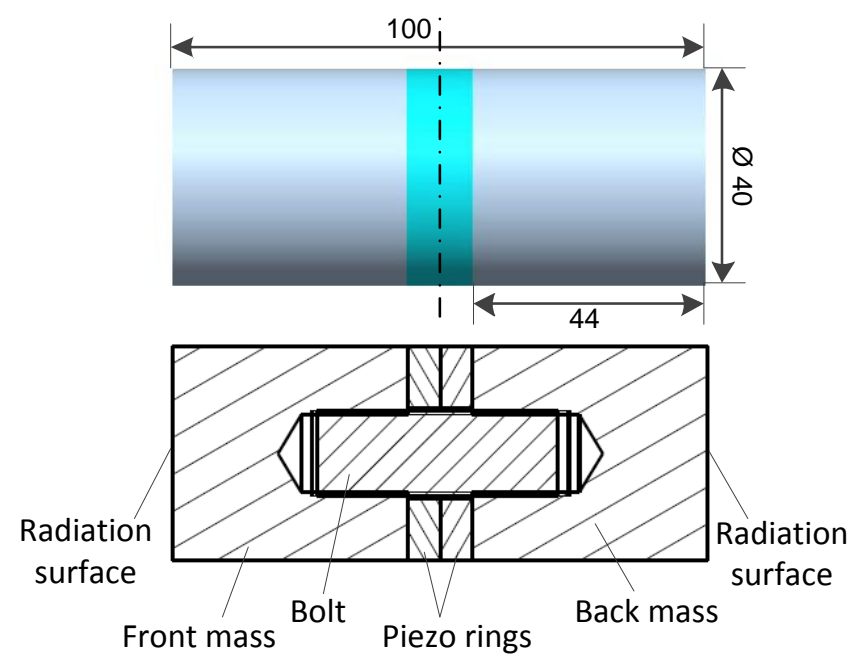

(a)

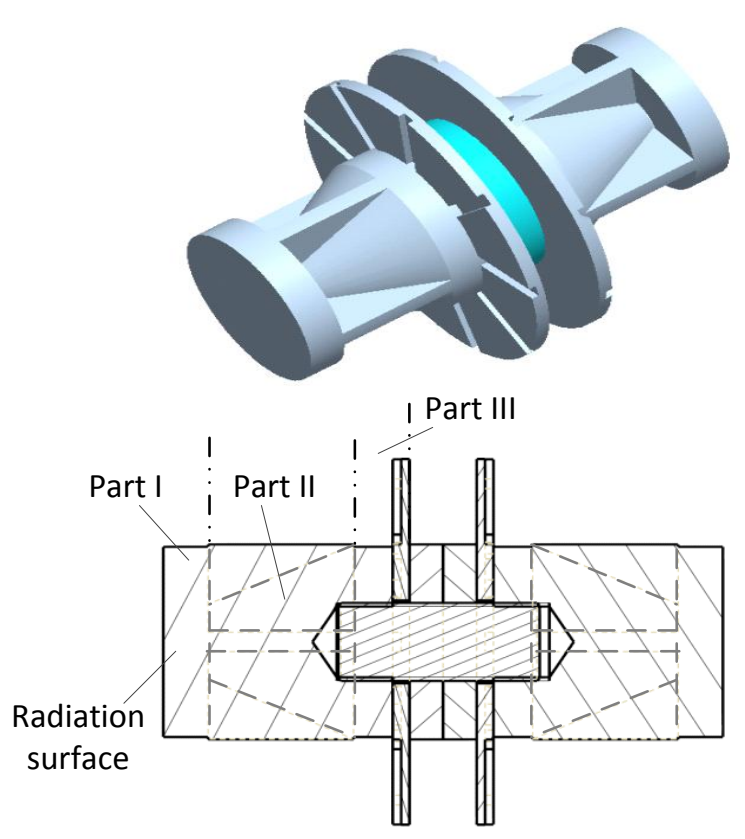

(b)

Fig. 1 


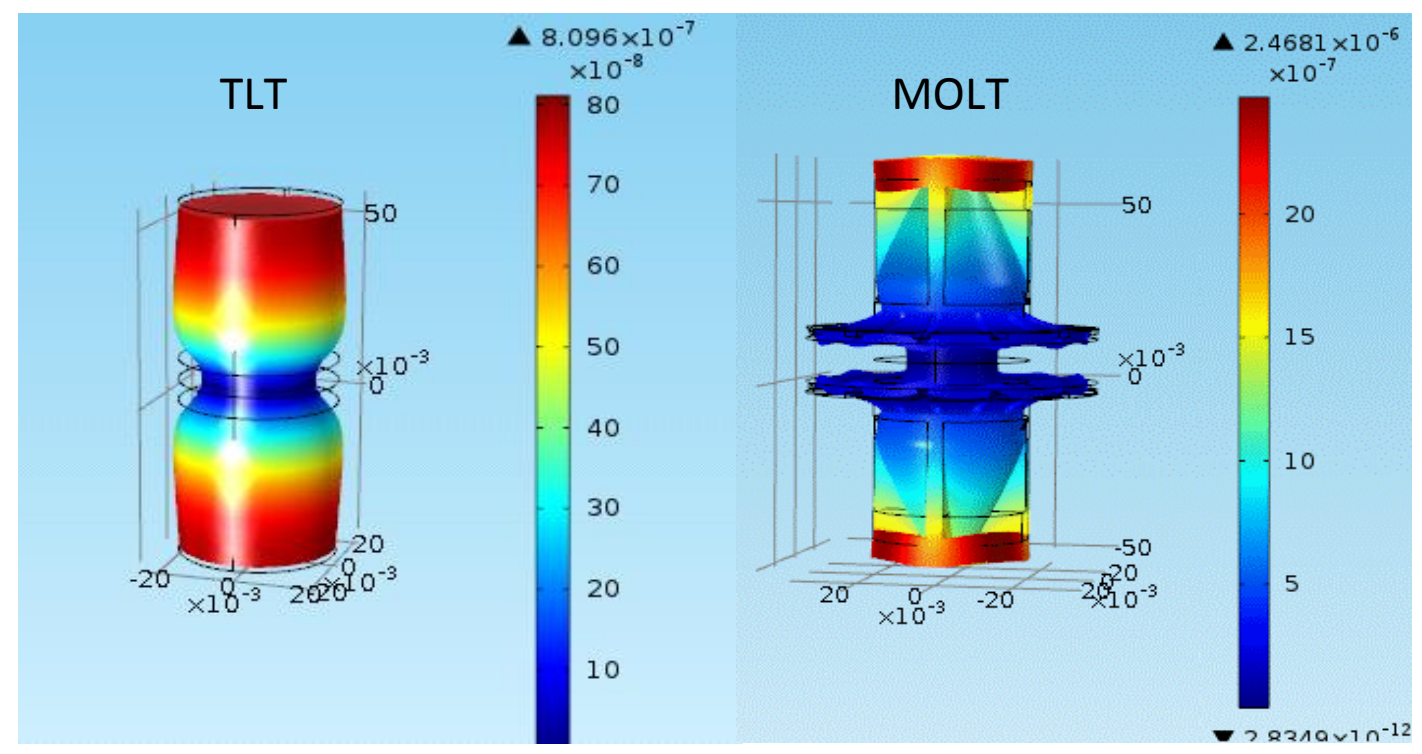

Fig. 2 


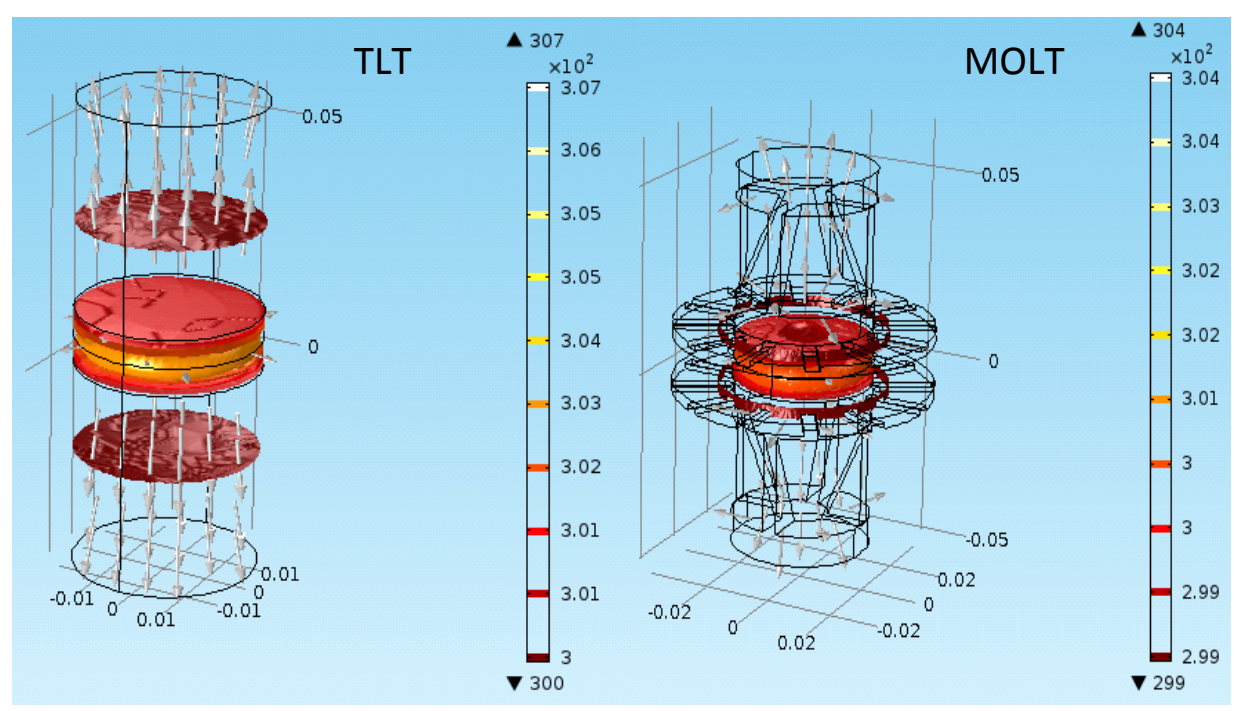

Fig. 3 


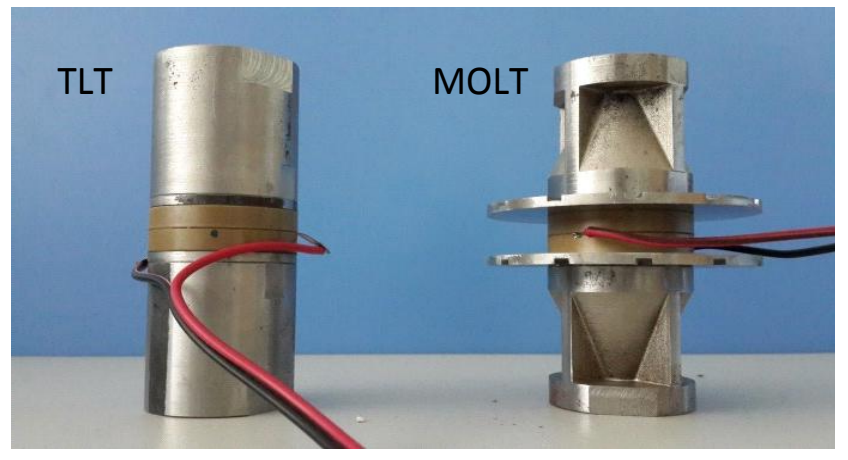

Fig. 4 


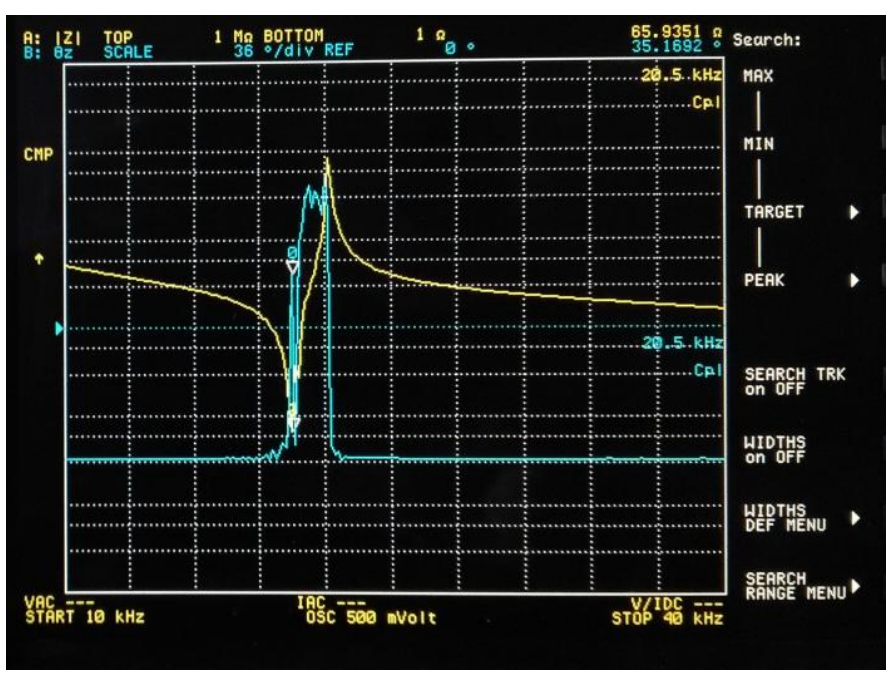

(a)

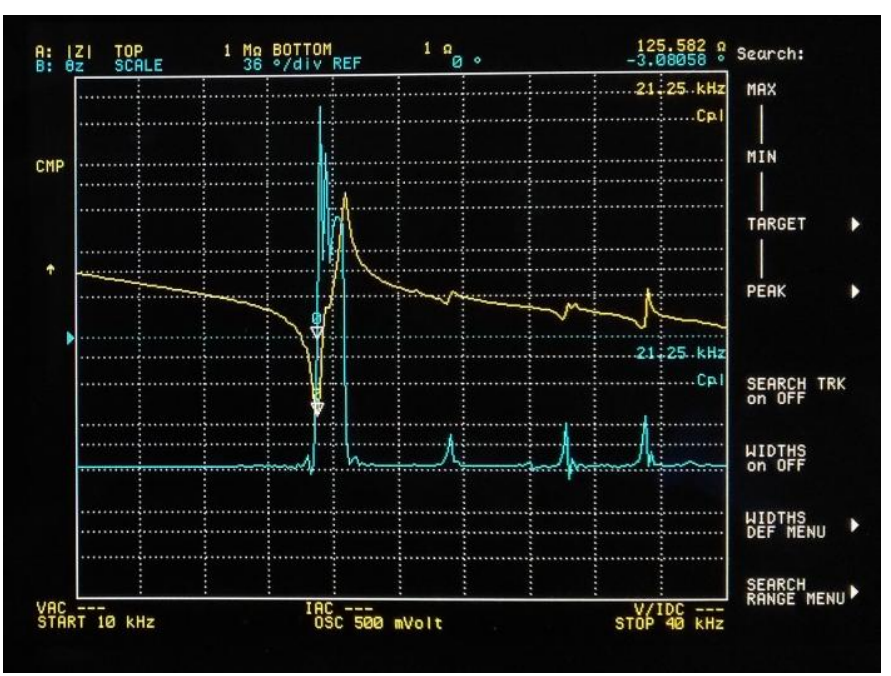

(b)

Fig. 5 


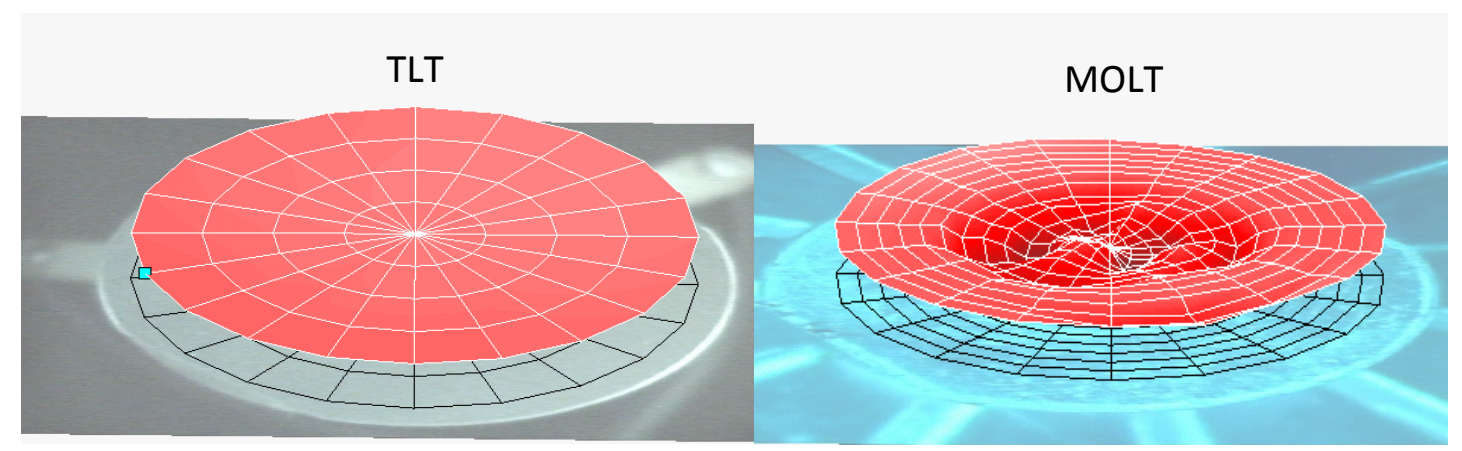

(a)

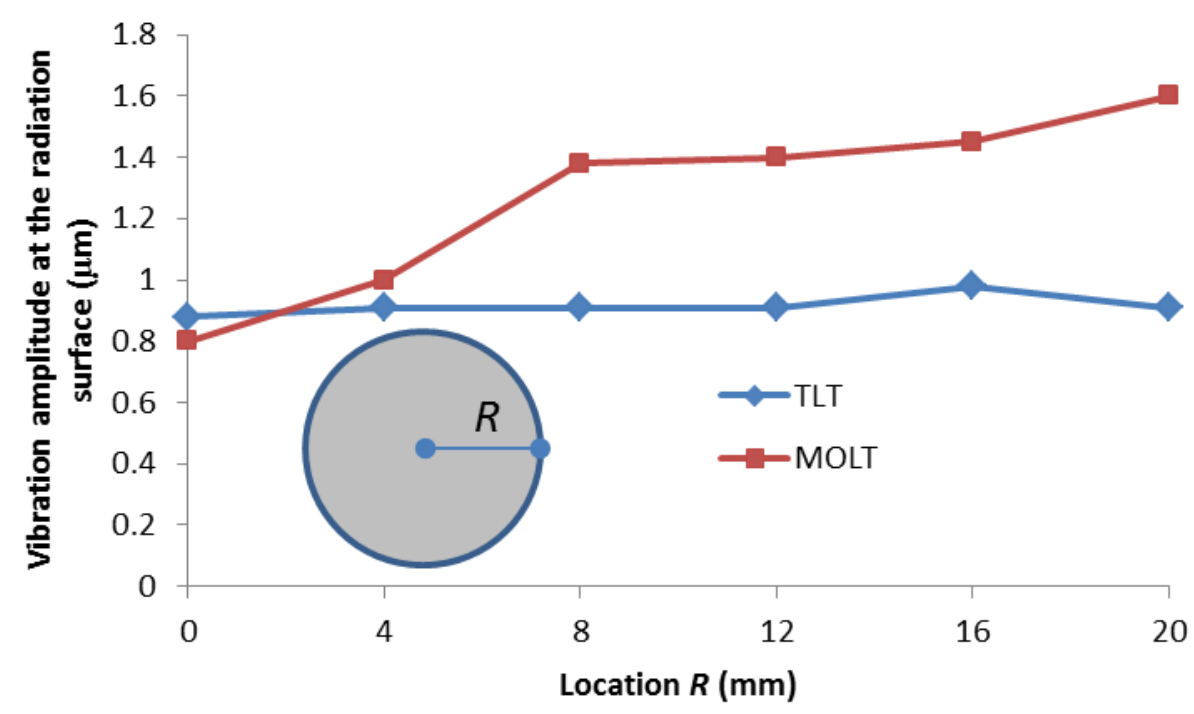

(b)

Fig. 6 


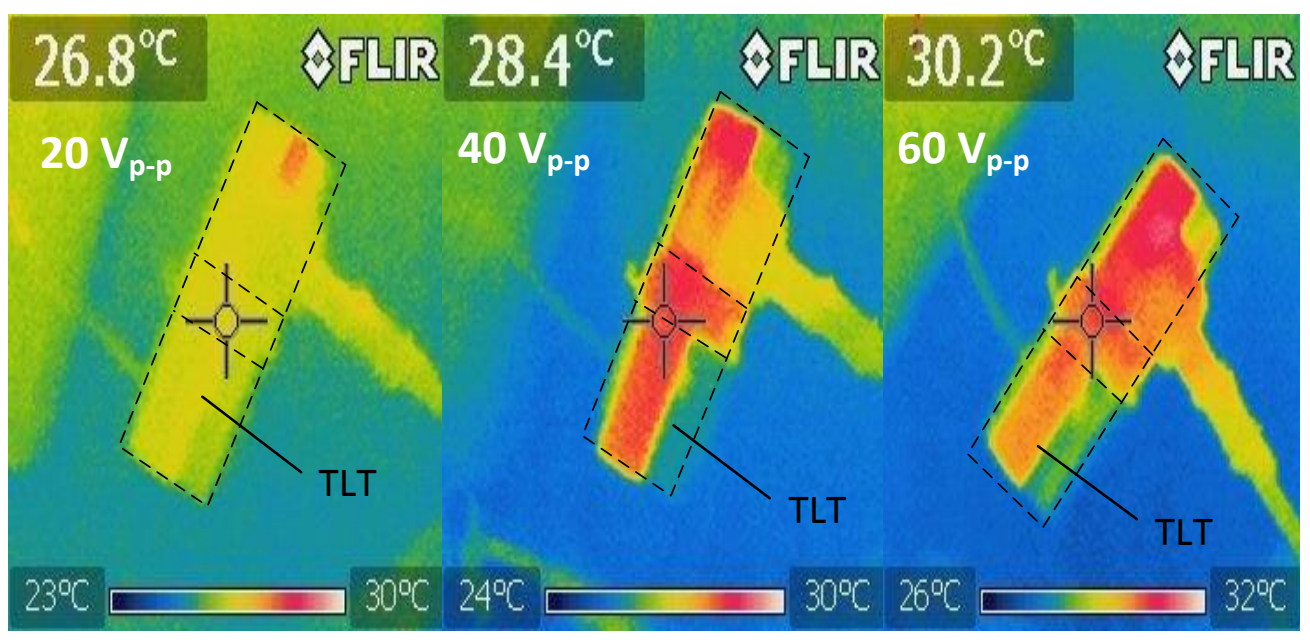

(a)

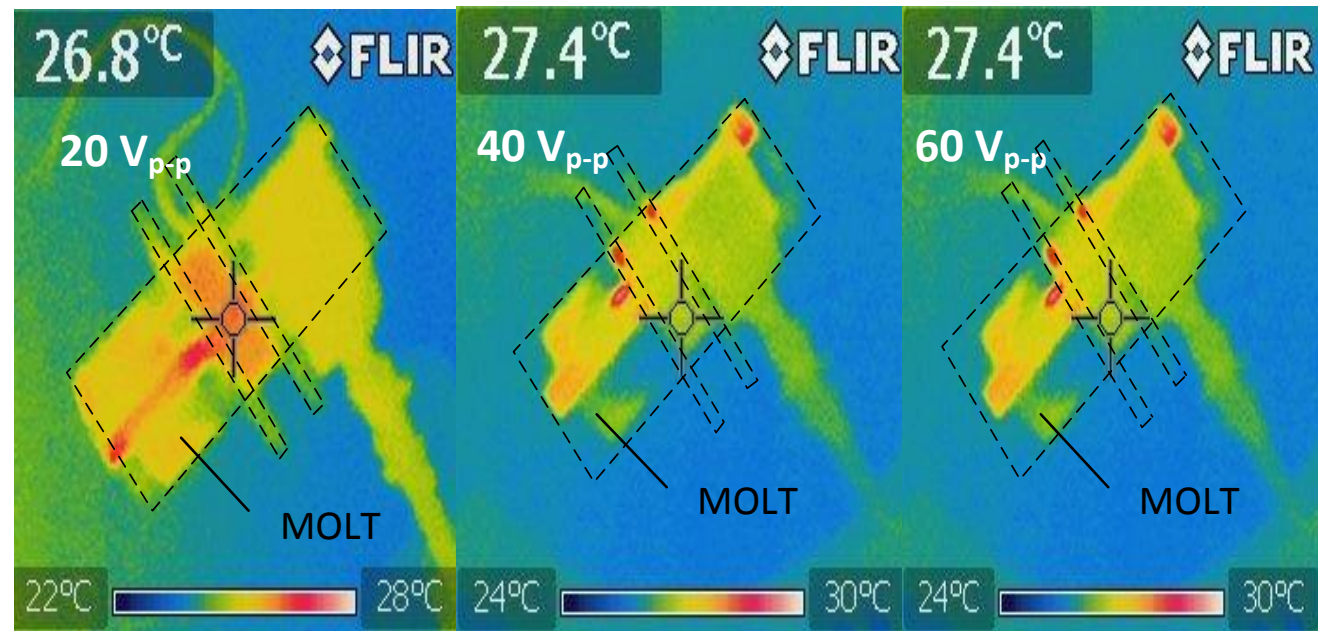

(b)

Fig. 7 


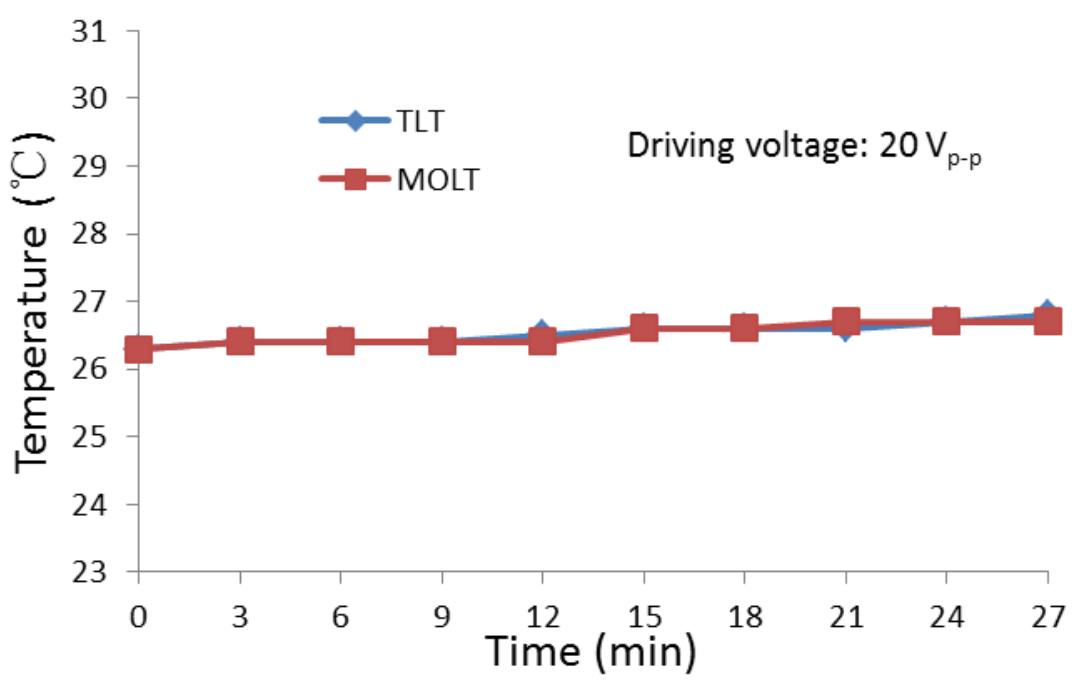

(a)

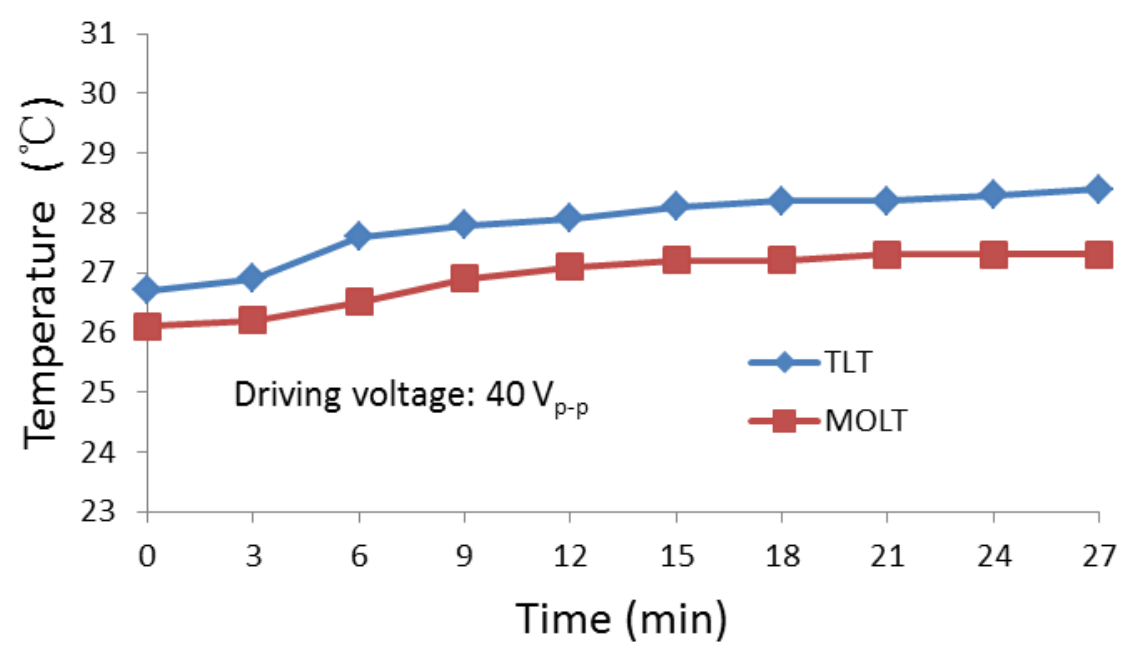

(b)

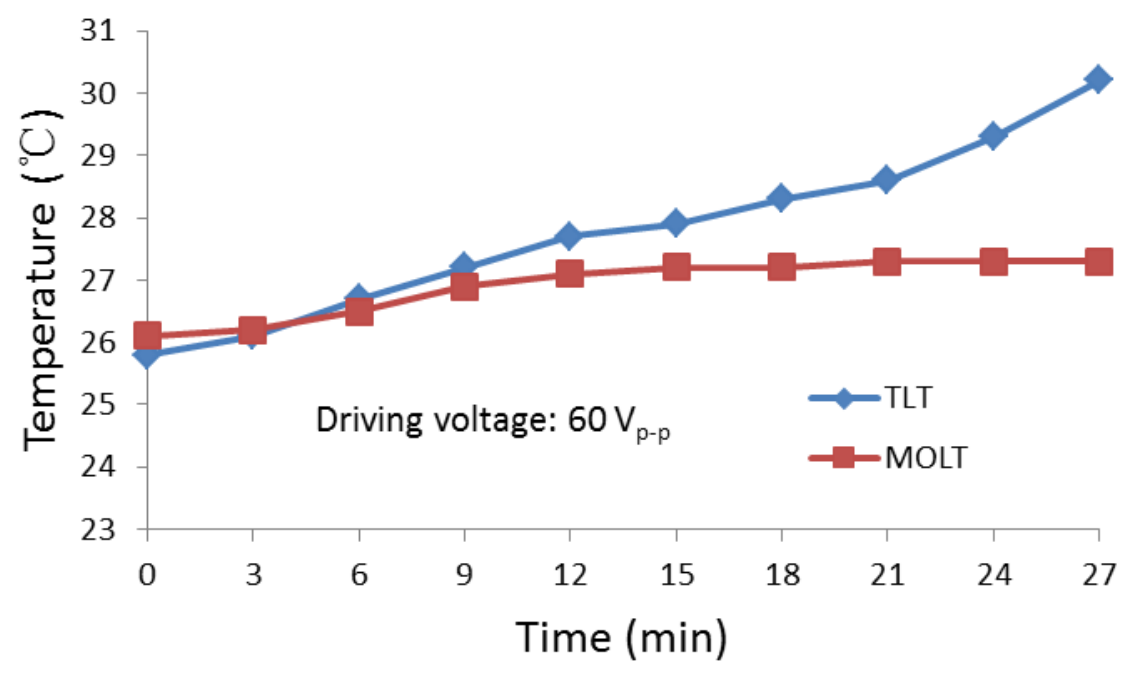

(c)

Fig. 8 


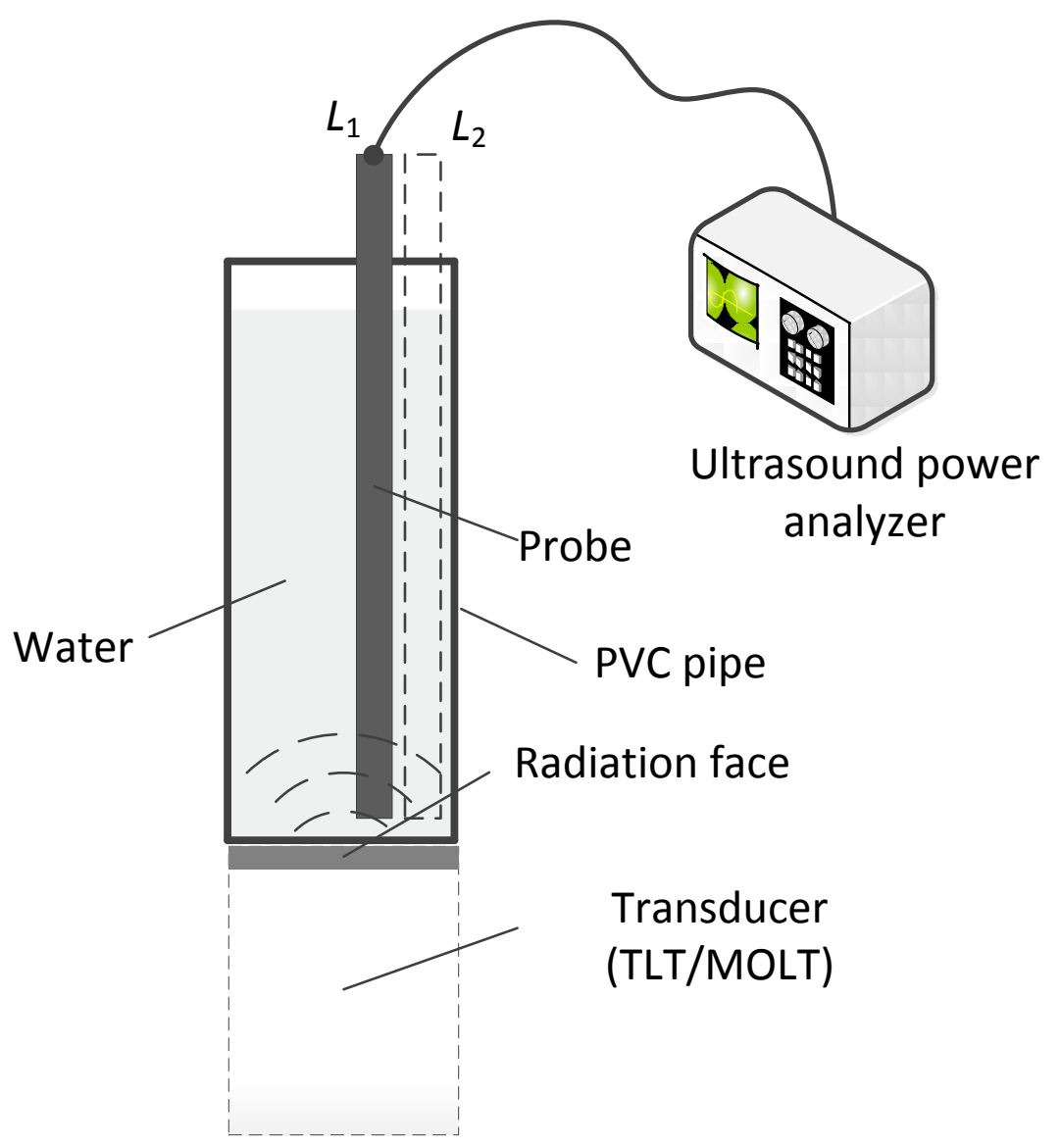

Fig. 9 


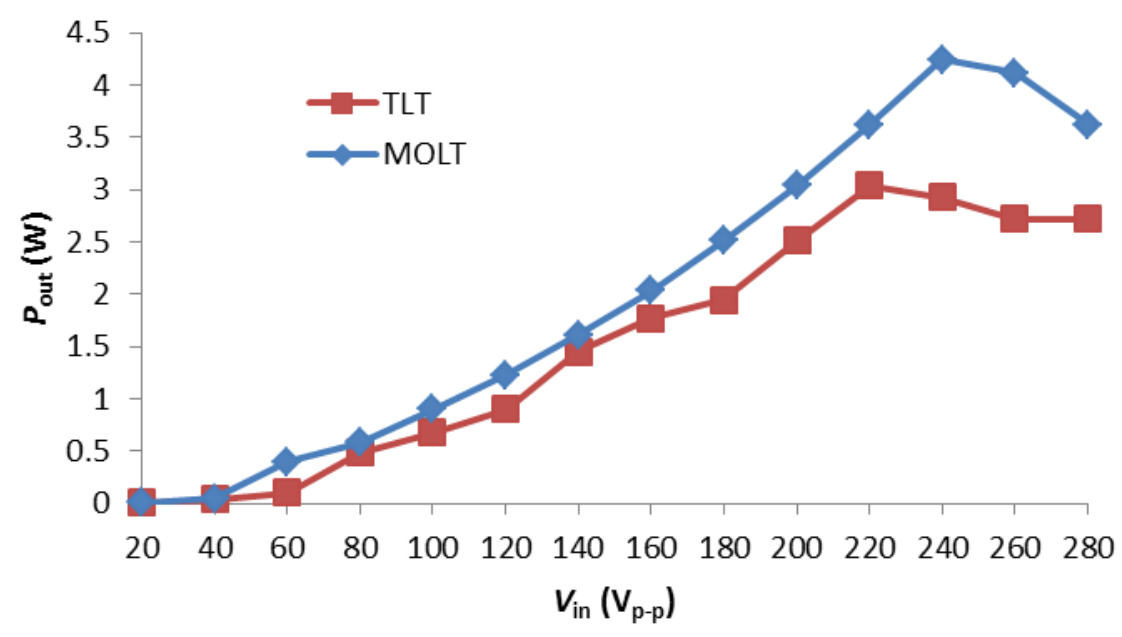

(a)

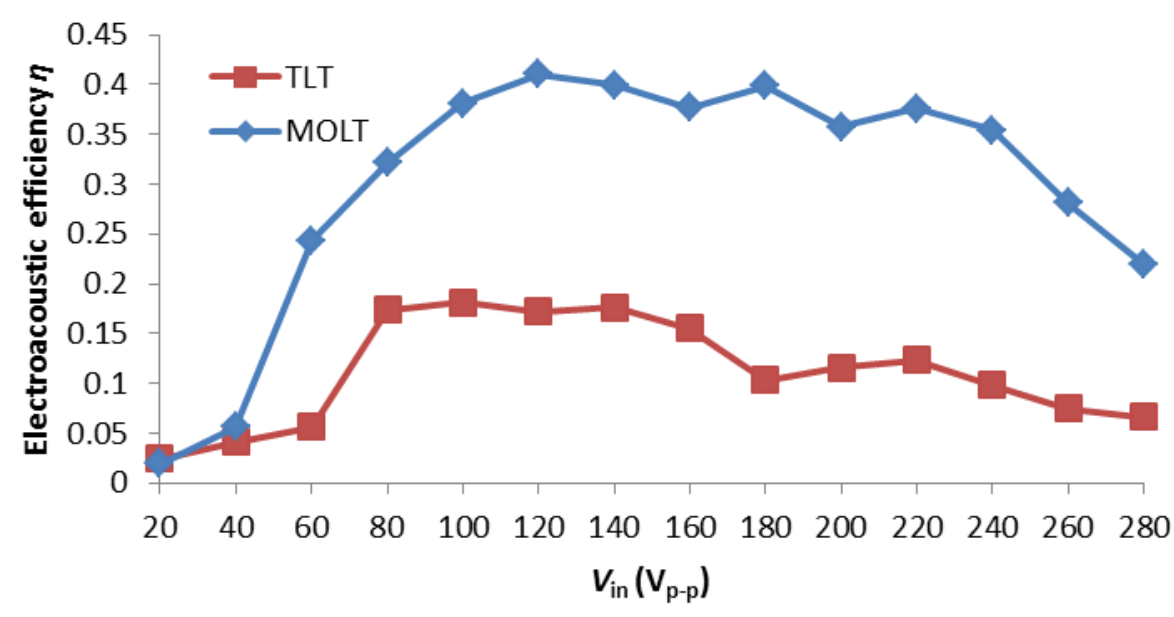

(b)

Fig. 10 